\title{
Evaluation of the role of combined prebiotic and probiotic supplements as prophylactic and therapeutic agents against experimental giardiasis
}

Original

Article

\author{
Yasmeen M Shaaban ${ }^{1}$, Zeinab R Hassan ${ }^{1}$, Rafiaa R Husseinn ${ }^{1}$, Aisha T Hassan ${ }^{1}$, Doaa \\ EA Salama ${ }^{2}$
}

Departments of Parasitology ${ }^{1}$ and Pathology ${ }^{2}$, Faculty of Medicine for Girls, Al-Azhar University, Cairo, Egypt

\begin{abstract}
Background: Giardiasis is considered as one of the most prevalent gastrointestinal parasitic infections worldwide. Most of the used anti-Giardia drugs may cause side effects and are often associated with clinical failure and drug resistance. Prebiotic and probiotic therapy has been posited as potential natural therapeutic agent for many parasitic diseases.

Objective: To evaluate the effect of combined prebiotic and probiotic therapy as a natural supplement and after addition to nitazoxanide (NTZ) in treatment of giardiasis in murine model.

Material and Methods: Ninety-six laboratory bred Swiss albino male mice were used. Mice were divided into three main groups that included 16 subgroups (6 mice each). Control group (A1-7) included control negative (non-infected non-treated), control positive (infected non-treated), and another five control subgroups (SGs) that received natural prebiotic and probiotic supplements and NTZ. Prophylactic group (B) included three SGs in which infected mice received prebiotic and probiotic separately and in combination for one week before infection and continued till the end of experiment. Infected treated group (C1-6) composed of six infected SGs that received treatment by prebiotic and probiotic separately, and combined prebiotic/probiotic, NTZ alone (full dose), and after addition of combined supplement to NTZ (half or full dose). Evaluation was achieved using parasitological, histopathological, immunohistochemical, and immunological parameters.

Results: There was significant reduction of Giardia cyst shedding in all prophylactic and infected treated SGs. The highest mean cyst reduction was observed in group C after addition of combined supplement to NTZ post infection (PI) and in group B receiving prophylactic combined supplement. Remarkable improvement of histopathological findings was observed especially in group $\mathrm{C}$ after receiving combined supplement in addition to NTZ, followed by other prophylactic and infected treated SGs. The results of immunohistochemical caspase 3 expression revealed that combined supplement was effective in preventing apoptotic cell death of intestinal cells that is induced by giardiasis especially after addition of combined supplement to NTZ in group C. Immunological assessment of interleukin-6 (IL-6) level showed significant increase in all treated SGs with boosted elevation in combined supplement after addition to NTZ in group C.

Conclusion: Combined prebiotic and probiotic products can be considered as good prophylactic and therapeutic agents when added to NTZ against giardiasis.
\end{abstract}

Keywords: caspase 3, giardiasis, interleukin 6, murine, nitazoxanide, prebiotic, probiotic.

Received: 5 July, 2021, Accepted: 13 August, 2021.

Corresponding Author: Zeinab R Hassan, Tel.: +20 1009659832, E-mail: dr_z37@yahoo.com

Print ISSN: 1687-7942, Online ISSN: 2090-2646, Vol. 14, No. 2, August, 2021.

\section{INTRODUCTION}

Giardia is one of the most prevalent intestinal flagellated protozoans that causes diarrhea in both children and adults ${ }^{[1]}$. Giardiasis has a worldwide distribution with a higher incidence rate in developing countries than developed ones and a higher infection rate in children than adults ${ }^{[2]}$. In certain localities in Egypt, the prevalence of giardiasis is up to $30.2 \%$, a fact that makes Egypt a hyper-endemic region according to the World Health Organization (WHO) criteria $^{[3]}$. Giardiasis is transmitted via the fecal-oral route through direct or indirect ingestion of cysts. Symptoms of infection vary from asymptomatic to acute steatorrhea, nausea, epigastric pain, and weight $\operatorname{loss}^{[1]}$. Post-infection complications may arise after Giardia elimination in the form of stunting, failure to thrive, chronic fatigue syndrome, irritable bowel syndrome, allergies and arthritis ${ }^{[4]}$.

The host defense against giardiasis includes both innate and adaptive immune responses that act in synchrony to control the infection. Innate immune mechanisms are the first line of defense against 
giardiasis. Adaptive immune mechanism requires mucosal humoral and cellular immune responses such as a balanced response of antigen-specific $\mathrm{CD} 4^{+}$ $\mathrm{T}$ cells, the release of cytokines, including ILs 4 and 6 , tumor necrosis factor (TNF)-a, and interferon (IFN)-c, and the production of specific IgA or IgG antibodies against parasite antigens ${ }^{[5,6]}$. The release of cathepsinlike cysteine proteases allows this parasite to attenuate local inflammatory responses ${ }^{[7]}$. One of the significant histopathological changes observed in giardiasis is a diffuse shortening of brush border microvilli. Villus atrophy and crypt hyperplasia was also reported ${ }^{[8]}$. Giardiasis induces this enteric pathology by activation of caspases that are the key mechanism of enterocytes apoptosis $^{[9]}$.

Among the forms of treatment for giardiasis, the use of chemotherapeutic drugs such as nitroimidazoles, furazolidone, paromomycin, benzimidazole compounds, NTZ as well as phototherapies are commonly highlighted ${ }^{[10]}$. However, due to an increase in resistance to these compounds, there is an urgent need for the development of new therapeutic strategies to fight the pathogen more healthily and effectively. Therefore, natural interventions and improvement of the intestinal microbiota through probiotic administration may be an important supplement for treatment ${ }^{[11]}$.

Probiotic refers to live microorganisms that have a beneficial effect on the health of the host when administered in suitable amounts ${ }^{[12]}$. Commensal microbiota and Giardia trophozoites compete for the same adhesion sites to colonize the small intestinal microenvironment. Additionally, supplementation of probiotic Lactobacilli (L.) strains showed antagonistic effects on $G$. duodenalis ${ }^{[13-16]}$. On the other hand, prebiotics are a group of nutrients that are degraded by gut microbiota. Their positive impacts on intestinal epithelial cell function include maintenance of metabolism, proliferation, differentiation and promotion of low $\mathrm{pH} 5$ of the gut environment, supporting microbiota and reduction in growth and viability of any pathogen ${ }^{[17,18]}$. Additionally, prebiotics supplementation was found to reduc the severity and duration of giardiasis ${ }^{[19]}$. Prebiotics may be used alone or added to probiotics to support them. The principal goal of this combination is to enhance the survival of probiotic microorganisms in the gastrointestinal tract ${ }^{[20]}$. Besides, prophylactic administration of probiotic Lactobacillus casei and prebiotic inulin together was assessed as anti-giardial agents in malnourished murine giardiasis ${ }^{[21]}$.

Therefore, the present study was designed to evaluate the effect of combined prebiotic and multistrain probiotics against giardiasis as a natural prophylactic agent and therapeutic when added to NTZ.

\section{MATERIAL AND METHODS}

This case control (experimental) study was conducted at Theodor Bilharz Research Institute (TBRI) and Parasitology Department, Faculty of Medicine for Girls (FMG), Al-Azhar University during the period from October 2019 to May 2021.

Study design: Mice were classified into three main groups; control (A), prophylactic (B), and infected treated (C). Groups were divided into 7, 3 and 6 SGs (6 mice each), respectively (Table 1). Animals infection was conducted on the $7^{\text {th }}$ day, and were sacrificed using intraperitoneal anesthesia after 30 days PI. Parasitological, histopathological, immunohistochemical, and immunological parameters were used to achieve study objective.

Experimental animals: Laboratory bred Swiss albino male mice (No. $=96$ ) weighing 20-25 g were divided as

Table 1. Classification of mice into study groups and subgroups.

\begin{tabular}{|c|c|}
\hline Groups and subgroups & Characteristics \\
\hline $\begin{array}{l}\text { Control group (A) } \\
\text { A1 } \\
\text { A2 } \\
\text { A3 } \\
\text { A4 } \\
\text { A5 } \\
\text { A6 } \\
\text { A7 }\end{array}$ & $\begin{array}{l}\text { Non-infected non-treated. } \\
\text { Infected non-treated. } \\
\text { Non-infected, received prebiotic. } \\
\text { Non-infected, received probiotic. } \\
\text { Non-infected, received combined prebiotic/probiotic. } \\
\text { Non-infected, received NTZ (full dose). } \\
\text { Non-infected, received NTZ (full dose) + combined prebiotic/probiotic. }\end{array}$ \\
\hline $\begin{array}{l}\text { Prophylactic group (B) } \\
\text { B1 } \\
\text { B2 } \\
\text { B3 }\end{array}$ & $\begin{array}{l}\text { Received prebiotic, then infected. } \\
\text { Received probiotic, then infected. } \\
\text { Received combined prebiotic/probiotic, then infected. }\end{array}$ \\
\hline $\begin{array}{l}\text { Infected treated group (C) } \\
\text { C1 } \\
\text { C2 } \\
\text { C3 } \\
\text { C4 } \\
\text { C5 } \\
\text { C6 }\end{array}$ & $\begin{array}{l}\text { Infected, received prebiotic PI. } \\
\text { Infected, received probiotic PI. } \\
\text { Infected, received combined prebiotic/probiotic PI. } \\
\text { Infected, received NTZ (full dose) PI. } \\
\text { Infected, received NTZ (full dose) + combined prebiotic/probiotic PI. } \\
\text { Infected, received NTZ (half dose) + combined prebiotic/probiotic PI. }\end{array}$ \\
\hline
\end{tabular}


6 mice for each of 16 SGs. Only parasite-free mice after stool examination were selected. The animals were provided and kept in TBRI Animal Producing Unit. They were kept also under specific pathogen-free conditions as well as lighting and temperature with free access to standard laboratory water and diet containing 24\% protein, $4 \%$ fat and about $4-5 \%$ fiber.

Mice infection: Stool samples were collected from patients complaining of steatorrhea attending the outpatient clinics at Alzahraa and Abu-Elrish hospitals. Samples were microscopically examined by direct wet mount (with and without iodine) ${ }^{[22]}$ to obtain positive samples that were further processed by washing with saline and centrifugation for preparation of Giardia cysts inoculum. The latter was counted and adjusted to infect mice orally with $10^{4}$ Giardia cysts using orogastric gavage according to Dyab et al. ${ }^{[23]}$. One week after infection, fecal pellets were collected daily and subjected to parasitological examination to ensure infection $^{[13]}$.

\section{Drugs and regimen dose}

- Commercial probiotic capsules were purchased from New Rhythm Comp. (New York, USA). They are composed of 25 billion multi-strain culture forming units (CFU/capsule). Each capsule is formulated with 20 of the most human probiotic strains ( $L$. acidophilus, L. rhamnosus, L. crispatus, L. plantarum, L. paracasei, L. bulgaricus, L. reuteri, L. casei, $L$. salivarius, L. helveticus, L. gasseri, Bifidobacterium (B.) lactis, B. bifidum, B. longum, B. breve, B. adolescentis, B. infantis, Leuconostoc mesenteroides, Lactococcus lactis, Streptococcus thermophiles). Every capsule was emptied in $2.5 \mathrm{ml}$ distilled water (DW) and was given in a dose of one billion CFU/mouse/day ${ }^{[13]}$. Each mouse received $0.1 \mathrm{ml} /$ day, i.e. one billion CFU/ day. Mice of SGs A4, A5, A7, B2, and B3 received the probiotic dose daily from the first day till the end the experiment (37 days). In infected treated SGs (C2, C3, $\mathrm{C5}$, and C6); mice received the probiotic dose daily from the $12^{\text {th }}$ day PI till the end of the experiment ( 22 days).

- Commercial prebiotic (Inulin) pure powder was purchased from Bulk Food Warehouse (Burlington, Canada). It was prepared daily by dissolving $200 \mathrm{mg}$ inulin powder in $10 \mathrm{ml} \mathrm{DW}$, and given in a dose of 2 $\mathrm{mg} / 0.1 \mathrm{ml} / \mathrm{mouse} /$ day $^{[19]}$. Mice of SGs A3, A5, A7, B2, and $\mathrm{B} 3$ received the prebiotic dose daily from the first day till the end the experiment (37 days). In infected treated SGs (C1, C3, C5, and C6), mice received the prebiotic dose daily from the $12^{\text {th }}$ day PI till the end of the experiment ( 22 days).

- Nitazoxanide was obtained from Utopia Pharmaceuticals (Cairo, Egypt). It was given orally as a suspension form at a full dose of $100 \mathrm{mg} / \mathrm{kg}$ body weight and a half dose of $50 \mathrm{mg} / \mathrm{kg}$ body weight for three consecutive days only ${ }^{[24]}$. Each tablet (500 $\mathrm{mg}$ ) was dissolved in $10 \mathrm{ml} \mathrm{DW}$. Mice treated with full NTZ dose received $2 \mathrm{mg} / 0.04 \mathrm{ml} /$ day, and those treated with half dose received $1 \mathrm{mg} / 0.02 \mathrm{ml} /$ day. It was given either alone or in addition to combined prebiotic and probiotic.

- Doses were dispensed by an oral tube fitted to an insulin syringe (100 units $/ \mathrm{ml}$ ), and given as 10 units for probiotic and prebiotic administration. For full and half doses of NTZ, 4 and 2 units were given respectively.

Parasitological examination: After administration of treatment, fecal pellets were collected at $12^{\text {th }}, 17^{\text {th }}$, $19^{\text {th }}$, and $30^{\text {th }}$ days $\mathrm{PI}^{[25]}$ and subjected to parasitological examination using the direct wet mount to count cyst number ${ }^{[22]}$ expressed per gram feces ${ }^{[26]}$. The percentage of reduction in parasite count $=[(a-b) / a] \times 100$, where $\mathrm{a}=$ mean number of parasites in the control group, and $\mathrm{b}=$ mean number of parasites in the treated group.

Histopathological examination: After sacrification of mice, segments of about one $\mathrm{cm}$ long from upper part of the small intestine were cut off and immediately fixed in $10 \%$ formalin then processed for paraffin embedding. All histopathological sections of $4 \mu \mathrm{m}$ thickness were stained with Hematoxylin \& Eosin stain $(\mathrm{H} \& \mathrm{E})^{[27]}$.

Caspase 3 immunohistochemistry ${ }^{[15,28]}$ : Four $\mu \mathrm{m}$ thickness of paraffin-embedded sections were used for immunohistochemical staining, using both polyclonal rabbit anti-active caspase- 3 as primary antibody and biotinylated goat anti-rabbit antibody as secondary antibody (DAKO, Carpinteria, California, USA). Standard positive and negative control sections were used for each assay. The represented fields of each section were randomly selected and interpreted in a blinded manner. The staining for caspase 3 was considered positive in the cytoplasm of intestinal epithelium. The intensity of staining and percentage of positive cells were estimated, then caspase 3 expression in intestinal tissue was evaluated according to $\mathrm{H}$ score as follows: $(-)$ : negative, $(+)$ : low or mild staining, $(++)$ : moderate staining, $(+++)$ : strong staining.

Immunological assessment: Blood was collected after mice sacrifice and centrifuged at $3000 \mathrm{rpm}$ for $5 \mathrm{~min}$. Sera were separated and stored at $-70^{\circ} \mathrm{C}$ until assessment. Boster immuonoleader mouse enzymelinked immunosorbent assay (ELISA) kit (Boster Biological Technology Co., Ltd.; California/USA) was used to quantify IL-6. Cytokine IL-6 level was calculated by comparing optical densities of tested sample to those of standard run in the same plate, values for standard curves and sample were calculated ${ }^{[29]}$.

Statistical analysis: Results were collected, tabulated and statistically analyzed using SPSS program version 16 for Microsoft Windows. Data were expressed as mean \pm standard deviation $( \pm S D)$ for quantitative 
variables and percent for qualitative variables. Means were compared using ANOVA test and values were considered significant at $P \leq 0.05$.

Ethical considerations: The protocol of this study was approved by the ethical committee of Faculty of Medicine for Girls Al-Azhar University and TBRI. All animals in the current study were handled in compliance with the international valid guidelines for animal experimental ethics.

\section{RESULTS}

Parasitological results: It was observed that Giardia cysts shedding in feces of all infected mice increased gradually with highest peak at $12^{\text {th }}$ day PI. In prophylactic (B) and infected treated (C) groups, there were significant reduction in the mean count of cyst shedding on all days (Tables 2 , and 3 ). The percentage of reduction in Giardia cysts shedding was higher in single probiotic administration (SGs: B2, and C2) than single prebiotic administration (SGs: B1, and C1), and the highest was in combined prebiotic and probiotic (SGs: B3, and C3). It was observed that the administration of NTZ full and half doses combined with prebiotic and probiotic (SG: C5 and C6) had the highest percentage of reduction in Giardia cysts shedding at all days PI (Fig 1).

Histopathological results: Histopathological examination of small intestine sections in the control non infected SGs (A1, A3, A4, A5, A6, and A7) that were represented in Fig. $(2, a-c)$ showed intact villi with normal villus/crypt ratio and preserved Goblet cells. Control positive, infected non treated SG (A2) showed sever destructive pathological effect of Giardia (Fig. 2d) and Giardia trophozoites between and attached to villi (Fig. 2e).

Improvement of the histopathological changes with varying degrees were observed in all prophylactics (Fig. 3a-c) as well as infected treated SGs (Fig. 4 a-f). Remarkable improvement of histopathological findings was observed in SG C5 (Fig. 4e) followed by SG B3 (Fig. 3c) and SG C6 (Fig. 4f).

Immunohistochemistry results: Caspase- 3 apoptotic activity showed strong expression in control positive (SG A2) (Fig. 5b). The peak of expression decreased with varying score in intestinal sections of all prophylactic and infected treated SGs (Fig. $5 \mathrm{c}-\mathrm{k}$ ). No apoptotic expression of caspase- 3 was observed in SG C5 treated with combined supplement in addition to NTZ (full dose) (Fig. 5j).

Table 2. The mean count and reduction $\%$ of Giardia cysts in all prophylactic SGs at the $17^{\text {th }}, 19^{\text {th }}$, and $30^{\text {th }}$ days PI.

\begin{tabular}{|c|c|c|c|c|c|c|}
\hline \multirow{3}{*}{$\begin{array}{c}\text { Prophylactic } \\
\text { SGs }\end{array}$} & \multicolumn{6}{|c|}{ Days (PI) } \\
\hline & \multicolumn{2}{|c|}{$17^{\text {th }}$} & \multicolumn{2}{|c|}{$19^{\text {th }}$} & \multicolumn{2}{|c|}{$30^{\text {th }}$} \\
\hline & Mean \pm SD & Reduction \% & Mean \pm SD & Reduction \% & Mean \pm SD & Reduction \% \\
\hline A2 & $52000 \pm 3535.5$ & -- & $24500 \pm 707.1$ & -- & $3500 \pm 353.6$ & -- \\
\hline B1 & $6875 \pm 2651.7$ & 87 & $4250 \pm 1060.7$ & 83 & $650 \pm 70.7$ & 82 \\
\hline B2 & $5500 \pm 707.1$ & 89 & $3600 \pm 548.5$ & 85 & $350 \pm 70.7$ & 90 \\
\hline B3 & $1500 \pm 141.4$ & 97 & $450 \pm 212.1$ & 98 & Zero & 100 \\
\hline \multicolumn{7}{|l|}{ Statistical analysis } \\
\hline ANOVA & \multirow{2}{*}{\multicolumn{2}{|c|}{$\begin{array}{c}677.8 \\
0000^{*}\end{array}$}} & \multicolumn{2}{|c|}{1471.5} & \multicolumn{2}{|c|}{458.1} \\
\hline$P$ value & & & \multicolumn{2}{|c|}{$0.000^{*}$} & \multicolumn{2}{|c|}{$0.000^{*}$} \\
\hline
\end{tabular}

A2: Infected non-treated; B1: Received prebiotic then infected; B2: Received probiotic then infected; B3: Received combined prebiotic/probiotic then infected. *: Significant $(P<0.05)$.

Table 3. The mean count and reduction $\%$ of Giardia cysts in all infected treated SGs at the $17^{\text {th }}, 19^{\text {th }}$, and $30^{\text {th }}$ days PI.

\begin{tabular}{|c|c|c|c|c|c|c|}
\hline \multirow{3}{*}{$\begin{array}{c}\text { Infected treated } \\
\text { SGs }\end{array}$} & \multicolumn{6}{|c|}{ Days (PI) } \\
\hline & \multicolumn{2}{|c|}{$17^{\text {th }}$} & \multicolumn{2}{|c|}{$19^{\text {th }}$} & \multicolumn{2}{|c|}{$30^{\text {th }}$} \\
\hline & Mean \pm SD & Reduction \% & Mean \pm SD & Reduction \% & Mean \pm SD & Reduction \% \\
\hline A2 & $52000 \pm 3535.5$ & -- & $24500 \pm 707.1$ & -- & $3500 \pm 353.6$ & -- \\
\hline C1 & $13000 \pm 4242.6$ & 75 & $10500 \pm 707.1$ & 57 & $700 \pm 141.4$ & 80 \\
\hline C2 & $7250 \pm 1060.7$ & 86 & $4250 \pm 353.6$ & 83 & $575 \pm 106.1$ & 84 \\
\hline C3 & $2800 \pm 1131.3$ & 95 & $550 \pm 424.2$ & 97.5 & $20 \pm 28.3$ & 99.5 \\
\hline C4 & $8500 \pm 2121.3$ & 84 & $150 \pm 70.7$ & 99 & $30 \pm 42.4$ & 99 \\
\hline C5 & $80 \pm 28.3$ & 99.5 & $60 \pm 56.6$ & 99.7 & Zero & 100 \\
\hline C6 & $1200 \pm 282.8$ & 98 & $90 \pm 14.1$ & 99.6 & Zero & 100 \\
\hline \multicolumn{7}{|l|}{ Statistical analysis } \\
\hline ANOVA & \multirow{2}{*}{\multicolumn{2}{|c|}{$\begin{array}{c}369.6 \\
0.000^{*}\end{array}$}} & \multirow{2}{*}{\multicolumn{2}{|c|}{$\begin{array}{l}2660.6 \\
0.000^{*}\end{array}$}} & \multicolumn{2}{|c|}{429.4} \\
\hline$P$ value & & & & & & \\
\hline
\end{tabular}

A2: Infected non-treated; C1: Infected, received prebiotic PI; C2: Infected, received probiotic PI; C3: Infected, received combined prebiotic/probiotic PI; C4: Infected, received NTZ PI; C5: Infected, received NTZ (full dose) + combined prebiotic/probiotic PI; C6: Infected, received NTZ (half dose) + combined prebiotic / probiotic PI. *: Significant $(P<0.05)$. 


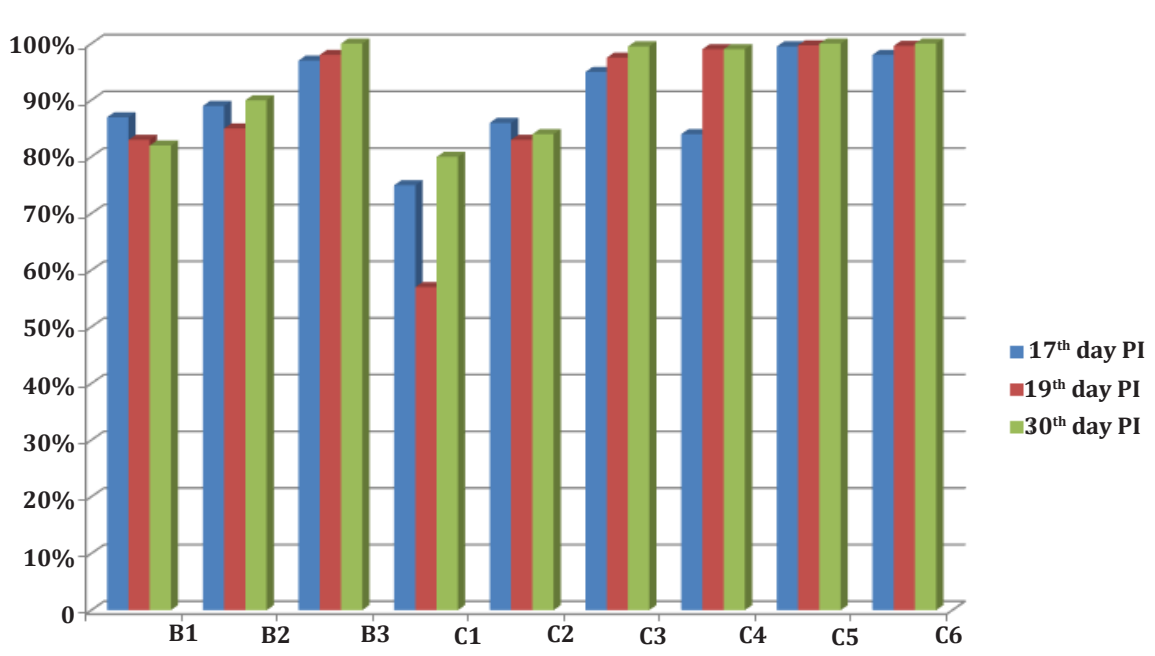

Fig. 1. Percentage of reduction in Giardia cysts shedding in different prophylactic and infected treated SGs at $17^{\text {th }}, 19^{\text {th }}$ and $30^{\text {th }}$ days PI.

B1: Prophylactic SG received prebiotic. B2: Prophylactic SG received probiotic. B3: Prophylactic SG received combined prebiotic+probiotic.

C1: Treated SG received prebiotic PI. C2: Treated DG received probiotic PI.

C3: Treated SG received combined prebiotic/probiotic PI.

C4: Treated SG received NTZ (full dose) PI.

C5: Treated SG received NTZ (full dose) + combined prebiotic/probiotic PI. C6: Treated SG received NTZ (half dose) + combined prebiotic/probiotic PI.

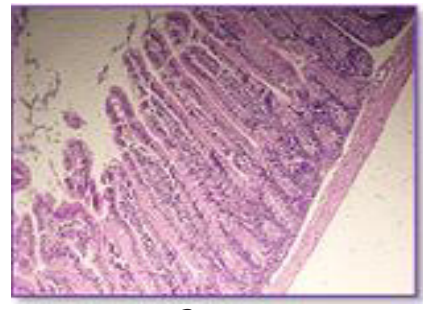

2a

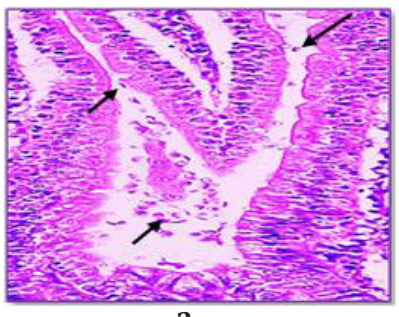

$2 \mathrm{e}$

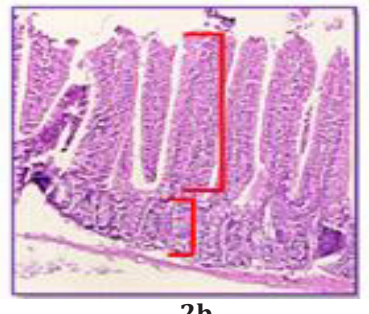

$2 \mathbf{b}$
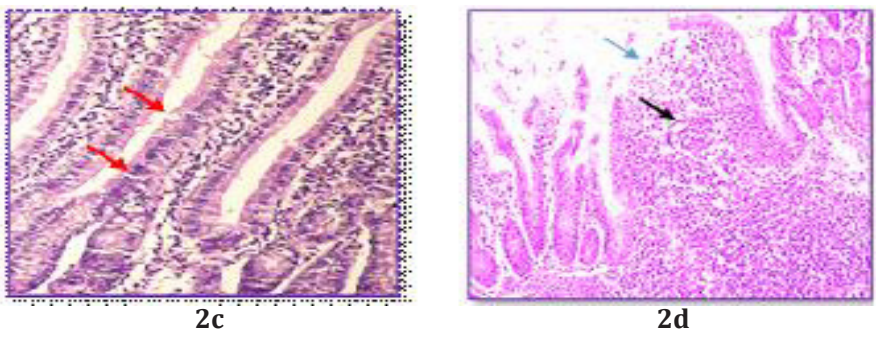

Fig. 2. Intestinal sections of mice in control group (A) stained with H\& E. 2a-2c: SGs A1, A3, A4, A5, A6, and A7 showed intact villi with normal villus/crypt ratio (red lines) and preserved Goblet cells (red arrow) (x100, x200). 2d: SG A2 showed marked villus shortening with expansion of villus core by excess inflammatory cellular infiltrate mainly of lymphocytes and plasma cells (black arrow), and focal villus epithelial necrosis with superficial ulceration (blue arrow) (x200). 2e: SG A2 showed Giardia trophozoites (arrows) (x400).

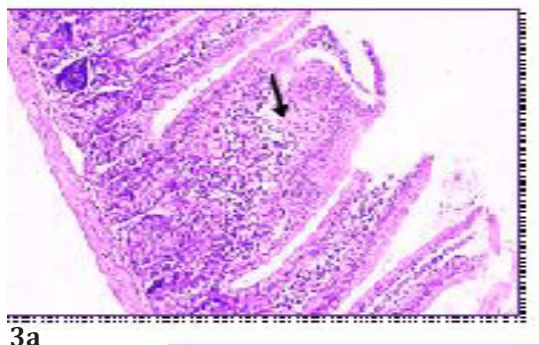

$3 a$

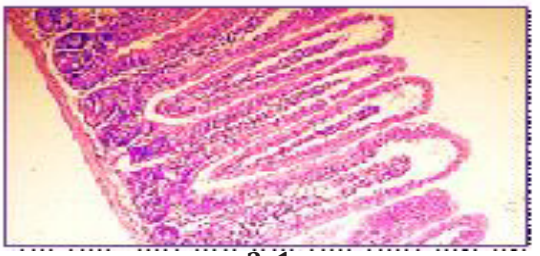

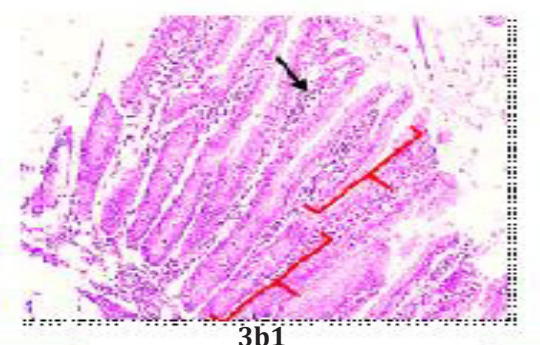
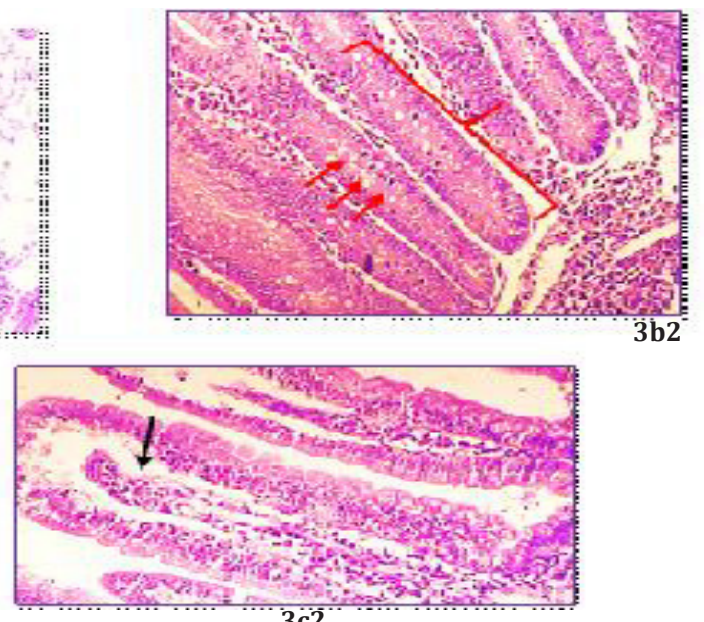

$3 \mathrm{c} 2$

Fig. 3. Intestinal sections of mice in prophylactic group stained with H\&E. 3a: SG B1 showed moderate villus atrophy and blunting with expansion by moderate mononuclear inflammatory cell infiltrate (arrow) (x100). 3b 1-2: SG B2 showed moderate widening of villi and expansion by mononuclear inflammatory cellular infiltrate (black arrow), crypt hyperplasia (red line) and increase mitotic activity (red arrows) (x100, 200x). 3c 1-2: SG B3 showed mild villus shortening with expansion by edema, mild inflammatory cellular infiltrate (black arrow) and no crypt hyperplasia (x100, x200). 

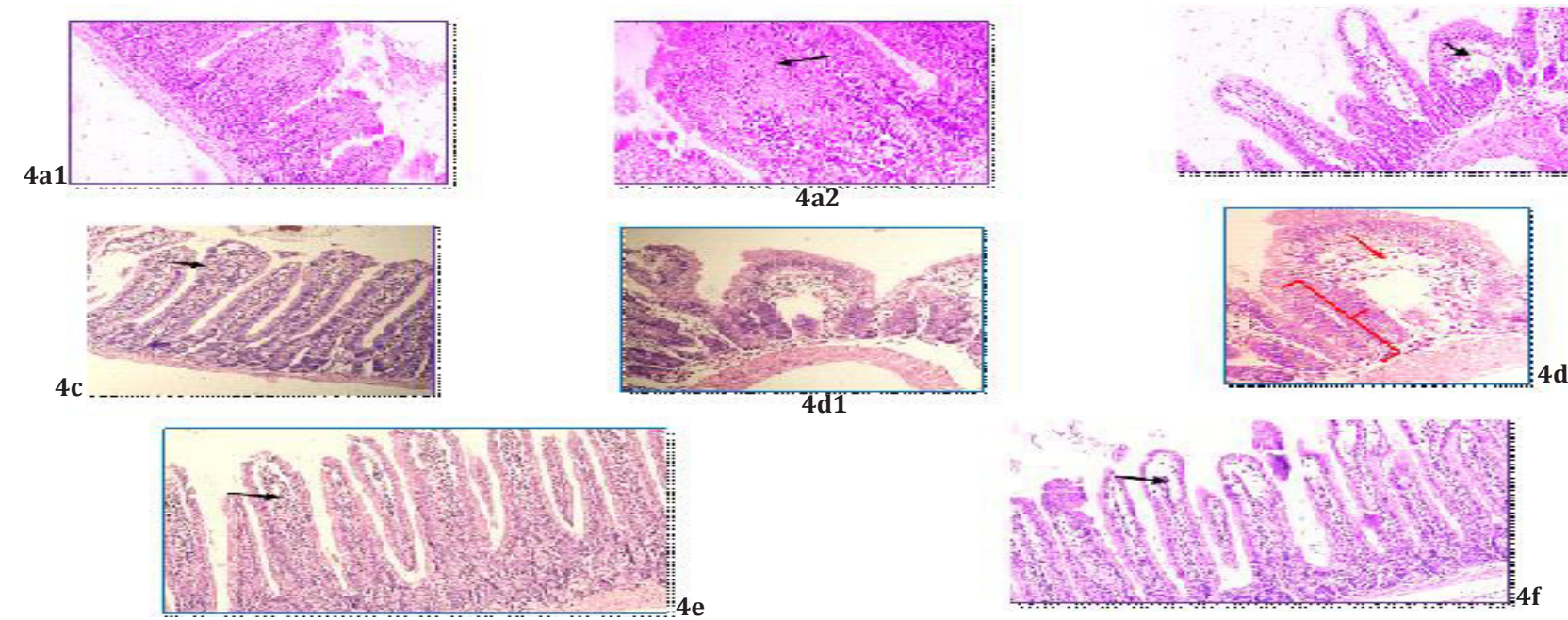

Fig. 4. Intestinal sections of mice in infected treated group stained with H\& E. 4a 1-2: SG C1 showed moderate villus atrophy and blunting with expansion by moderate mononuclear inflammatory cellular infiltrate and focal villus necrosis in villi (arrow) (x100, x200). 4b: SG C2 showed moderate villus shortening and blunting (arrow) with expansion of villus core by edema and moderate inflammatory cellular infiltrate (x100). 4c: SG C3 showed mild villus shortening with expansion by edema, mild inflammatory cellular infiltrate (arrow) (x100). 4d 1-2: SG C4 showed moderate villus shortening and blunting with expansion of villus core by edema, moderate inflammatory cellular infiltrate (red arrow) and crypt hyperplasia (red lines) (x100, x200). 4e: SG C5 showed intact villi, normal crypt/ villus ratio with mild inflammatory cellular infiltration (arrow) (x100). 4f: SG C6 showed mild villus shortening with expansion by edema and inflammatory cellular infiltrate (arrow) (x100).
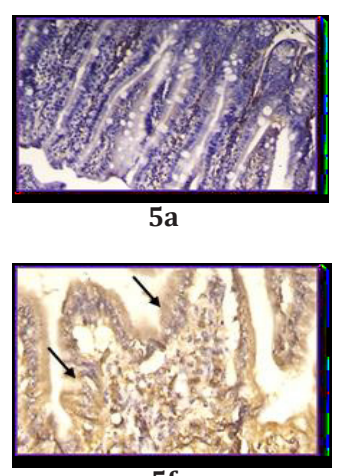

$5 f$

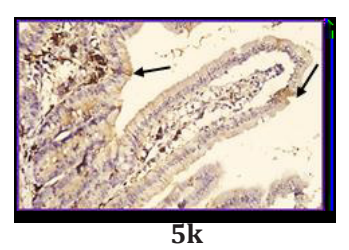

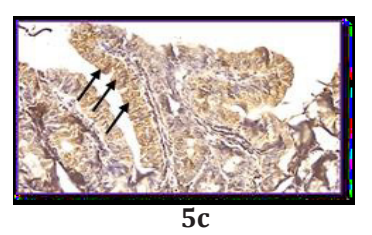

$5 c$
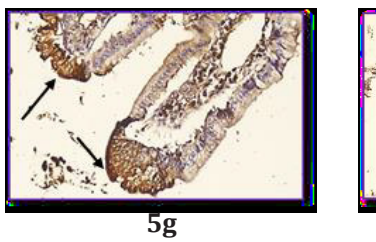
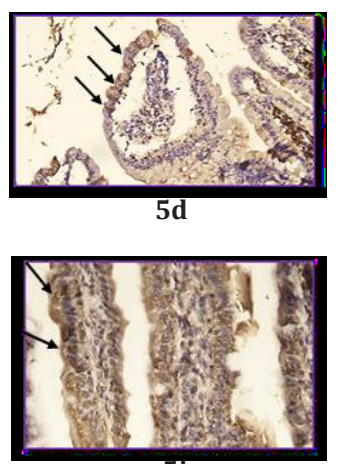

$5 \mathbf{i}$
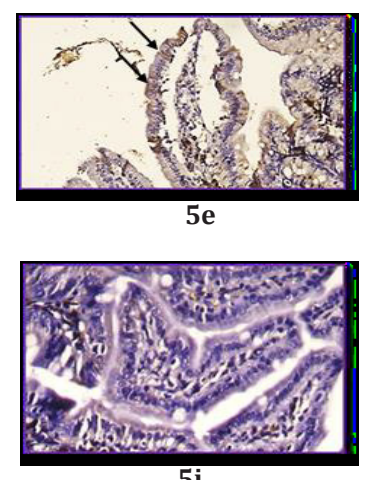

$5 j$

Fig. 5. Intestinal sections of mice (immunohistochemical staining for caspase-3). 5a: SG A1 showed negative expression (-) (x200). 5b: SG A2 showed brown cytoplasmic staining in intestinal epithelium, strong expression (+++) (arrows) (x400). 5c: SG B1 showed moderate expression (++) (arrows) (x200). 5d: SG B2 showed mild expression (+) (arrows) (x200) 5e: SG B3 showed mild expression (+) (arrows) (x200). 5f: SG C1 showed moderate expression (++) (arrows) (x400). 5g: SG C2 showed moderate expression (++) (arrows) (x400). 5h: SG C3 showed mild expression (+) (arrow) (x200). 5i: SG C4 showed moderate expression (++) (arrows) (x400). 5j: SG C5 showed negative expression (-) (x400). 5k: S.G.C6 showing mild expression (+) (arrow) (x200).

Immunological assessment of IL6: The results showed significant increase in the mean level concentrations of IL-6 in all prophylactic (B) and infected treated (C) mice (Table 4).

Table 4. Comparison between means \pm SD of serum IL-6 cytokine in different SGs.

\begin{tabular}{lrcc}
\hline \hline & \multicolumn{1}{c}{ IL-6 } & \multicolumn{2}{c}{ Statistical analysis } \\
\cline { 2 - 4 } & Mean \pm SD & ANOVA & P value \\
\hline A1 & $5.70 \pm 0.57$ & & \\
B1 & $16.10 \pm 0.28$ & & \\
B2 & $16.85 \pm 0.21$ & & \\
B3 & $18.75 \pm 0.49$ & & \\
C1 & $12.60 \pm 0.71$ & & \\
C2 & $14.15 \pm 0.35$ & $\mathbf{2 5 4}$ & \\
C3 & $16.15 \pm 0.92$ & & \\
C4 & $16.65 \pm 0.78$ & & \\
C5 & $20.95 \pm 0.92$ & & \\
C6 & $19.15 \pm 0.35$ & & \\
A2 & $10.35 \pm 0.07$ & & \\
\hline \hline
\end{tabular}

A1: Non-infected non-treated; A2: Infected non-treated; B1: Received prebiotic then infected; B2: Received probiotic then infected; B3: Received combined prebiotic/probiotic then infected; C1: Infected, received prebiotic PI; C2: Infected, received probiotic PI; C3: Infected, received combined prebiotic/probiotic PI; C4: Infected, received NTZ (full dose) PI; C5: Infected, received NTZ (full dose) + combined prebiotic/ probiotic PI; C6: Infected, received NTZ (half dose) + combined prebiotic/probiotic PI. *: Significant $(P<0.05)$. 


\section{DISCUSSION}

In the present study, prophylactic prebiotic and probiotic treatment (group B) prior to Giardia infection was done to evaluate the effect of infection if it happens in the presence of healthy intestinal flora. Shukla et al. ${ }^{[19]}$ reported that prebiotic inulin supplementation even to malnourished Giardiainfected mice reduced both cyst and trophozoite counts. The reduction in the severity of giardiasis after prebiotic supplementation was explained as due to increase of Lactobacilli survival and colonization in the gut. The results of probiotics alone as an antiGiardia agent in the present study are in accordance with another report by Humen et $a l^{[30]}$ who demonstrated that in animals receiving probiotic for seven days before Giardia inoculation, the intensity and time of infection were reduced. Similar results were reported ${ }^{[25]}$. Additionally, Shukla et al. ${ }^{[31]}$ reported that after supplementation by L. casei, the experimentally infected mice became Giardia free by day 30 PI.

Our study demonstrated significant mean count of Giardia cysts reduction in both prophylactic (B) and infected treated (C) groups on the $17^{\text {th }}, 19^{\text {th }}$ and $30^{\text {th }}$ days PI. Combined prebiotic and probiotic supplement in either of prophylactic or infected treated groups, generally gave higher percentage of reduction than NTZ alone. The highest percentage of reduction was observed when combined prebiotic and probiotic was added to NTZ (full dose), followed by combined prebiotic and probiotic added to NTZ (half dose), then the prophylactic combined prebiotic and probiotic SG.

Amer et al. ${ }^{[32]}$ added that reduced parasite density both in vitro and in vivo occurred after administration of bacteriocins derived from newly isolated Egyptian strains of probiotic Lactobacilli. In a similar study, Mazroue et $a l^{[15]}$ registered a significant reduction of the excreted Giardia cysts count in prophylactic and infected treated probiotic SGs. It was postulated that the probiotic anti-Giardia effect may be due to interference with attachment of Giardia trophozoites to the mucosa, competition for nutrients and production of antimicrobial compounds ${ }^{[33]}$.

In a metanalysis study, Ventura et al. ${ }^{[34]}$ recorded the anti-giardial effect of different probiotic strains. Besides, Perrucci et al. ${ }^{[16]}$ stated that commercial multistrain probiotic Slab51(containing 200 billion lactic acid bacteria) showed higher antagonistic effects on G. duodenalis both in vitro and ex vivo intestinal tissue cultures. Similarly, Sand et al. ${ }^{[35]}$ reported a high therapeutic efficacy of potent commercial Acidopillus capsules (containing 50 million live probiotic bacteria of five species) against experimental giardiasis. In the present study, commercial multi-strain probiotics gave potent efficacy that may be explained by the synergistic effect of different probiotic strains used. The fact that prebiotics, probiotics, and symbiotics can be used as preventive and therapeutic agents for many human diseases was previously declared ${ }^{[36]}$. Their efficacy depends on the etiology of the disease and introducing the suitable probiotic strain to restore the balance of intestinal flora.

The significant reduction in cyst shedding after combined prebiotic and probiotic supplement in the present study agreed with the results of Shukla et al. $^{[21]}$ who found that prophylactic administration of synbiotic ( $L$. casei + inulin) to malnourished Giardia-infected mice led to increase body mass, small intestine mass, Lactobacilli counts, and reduced severity of giardiasis that was evident by decreased cyst and trophozoite counts. However, we recorded the highest reduction in cyst shedding after addition of NTZ to combined prebiotic and probiotic treatment, which implies that the most beneficial way to treat giardiasis might be through combination of nutritional interventions and chemotherapeutic agents $^{[37]}$. This was confirmed by the combination of Saccharomyces boulardii with metronidazole to clear all the symptoms related to giardiasis ${ }^{[38]}$. Moreover, Shukla et $a l^{[39]}$ observed the synergistic effect of co-administration of probiotic and albendazole, tinidazole, metronidazole, and NTZ to Giardiainfected mice, which resulted in reduced Giardia cysts shedding in feces and trophozoite numbers in the intestinal fluid. Prophylactic and treated mice with both L. casei and metronidazole SGs showed the highest shedding reduction of Giardia cysts $^{[15]}$. It is worth mentioning that NTZ was approved for the treatment of giardiasis in humans by the FDA ${ }^{[40]}$ and can be given as a potential alternative therapy for metronidazole-resistant giardiasis ${ }^{[41]}$. Accordingly, it was chosen as chemotherapeutic agent in the present study.

The normal intestinal architecture demonstrated in our histopathological sections of control non infected non treated mice was similarly recorded in the supplemented and NTZ control SGs. In reverse the control positive infected mice revealed marked histopathological changes which agreed with several researches reporting marked villus shortening, necrosis and ulceration with excess inflammatory cellular infiltrates as a destructive effect of Giardia $^{[8,13,15,19,35]}$.

It was also observed that supplementation of either prebiotic or probiotic to infected mice helped in partial improvement of the histopathological changes which was better in prophylactic (B) than infected treated (C) groups and more specifically in probiotic treated SGs (B2, C2). These results agreed with the previous observation ${ }^{[19]}$ of reduced mucosal damage in microvilli with prebiotic supplementation either prior to or simultaneously with experimental 
Giardia infection. Also, many authors reported that administration of probiotics to Giardia infected mice reduced atrophy of villi and cellular infiltration ${ }^{[8,13,15,35]}$. In the present study, remarkable improvement in the histopathological changes with mild villus atrophy was noticed with combined prebiotic and probiotic supplementation either in prophylactic (B3) or infected treated mice (C3). Similar results were observed after addition of combined supplement to NTZ (half dose) (C6). While addition of combined supplement to NTZ (full dose) (C5) helped in restoring the normal mucosal architecture with normal villus to crypt ratio, although mild inflammation in the lamina propria was still noticed.

Shukla et al. $^{[21]}$ observed that supplementation of synbiotic (L. casei + inulin) to malnourished-Giardia infected mice helped in better improvement of the affected mucosal layers. Restoring nearly normal villus architecture in Giardia-infected mice after addition of probiotic to chemotherapeutic agents was confirmed after co-administration of probiotic and albendazole ${ }^{[39]}$, and also in mice treated with both $L$. casei and metronidazole ${ }^{[15]}$.

Concerning the immunohistochemical results of intestinal sections, caspase-3 apoptotic activity expression was strong in control positive infected non-treated (A2) mice which agreed with previous observations ${ }^{[16,28]}$. Prebiotic and probiotic supplement in the present study, reduced apoptotic caspase-3 expression with varying scores in all prophylactic and infected treated SGs. Reduction in the peak of caspase-3 expression was documented in ex vivo intestinal tissue cultures after $18 \mathrm{~h}$ of incubation with probiotic Slab51 ${ }^{[16]}$. On the other hand, in the present study treatment with combined supplement in addition to full dose NTZ (C5) revealed no apoptotic expression of caspase- 3 which agreed with the corresponding histopathological result of the same SG (C5) in our present study. This proves that combinations of these supplements with specific treatment are promising for enterocytes protection against giardiasis.

Some cytokines, such as IL-6, IL-12 and IL-17, can activate both innate and adaptive pathways ${ }^{[22-45]}$. IL-6 released by mast cells, dendritic cells or T cells, is a key regulator of B-cell maturation and promotes antibody class switching to produce $\operatorname{Ig} \mathrm{A}^{[45,46]}$. It was recorded that IL-6-deficient mice were unable to eradicate Giardia and had altered intestinal cytokine responses despite having normal levels of Ig ${ }^{[42,47]}$. Our present study demonstrated significant increase in serum IL-6 level in all prophylactic and infected treated groups. The highest IL-6 level was observed in full dose NTZ combined with prebiotic and probiotic (C5), followed by half dose NTZ combined with prebiotic and probiotic (C6), then prophylactic combined prebiotic and probiotic (B3). The results of the present study concur with those of research that revealed modulation of both non-specific and specific immune responses. This modulation was manifested by increased nitric oxide, secretory anti-Giardia IgA and IgG antibody and antiinflammatory cytokines (ILs 6, and 10) levels, after separate supplementation of either prebiotic inulin ${ }^{[19]}$ or probiotic L. casei ${ }^{[34,48,49]}$, and also with combined supplements ${ }^{[21]}$ either prior to or simultaneously with Giardia infection to nourished or malnourished mice.

In conclusion combined prebiotic and probiotics (multi strains) have demonstrated a significant potential supplement for medication of giardiasis. Hence, combined products can be considered as successful prophylactic and therapeutic agents when added to NTZ for treatment of giardiasis.

Acknowledgement: Sincere thanks to the staff members of the Clinical pathology Department, Faculty of Medicine, Al-Azhar University for their help in collection of the samples.

Author contribution: Shaaban YM and Hassan ZR designed the experiment, collected the samples, performed experimental infection, parasitological examination, immunological assessment and wrote the manuscript. Salama DEA performed histopathological and immunohistochemical assessment. Hassan AT and Hussein RR supervised the work and shared in writing the manuscript.

Conflict of interest: The authors declare that there is no conflict of interest.

Financial statement: No financial support was provided.

\section{REFERENCES}

1. Hooshyar H, Rostamkhani P, Arbabi M, Delavari M. Giardia lamblia infection: review of current diagnostic strategies. Gastroenterol Hepatol Bed Bench 2019; 12(1):3-12.

2. Zajaczkowski P, Mazumdar S, Conaty S, Ellis JT. Epidemiology, and associated risk factors of giardiasis in a peri-urban setting in New South Wales Australia. Epidemiol Infect 2018; 28:1-9.

3. El-Badry AA, Mohammed AF, Abdul Gawad E. Predominance of Giardia intestinalis assemblage B in diarrheic children in Sharkia, Egypt. PUJ 2017; 10: 39-43.

4. Hanevik K, Wensaas KA, Rortveit G, Eide GE, Mørch K, Nina L. Irritable bowel syndrome and chronic fatigue 6 years after Giardia infection: a controlled prospective cohort study. Clin Infect Dis 2014; 59(10):1394-1400.

5. Langford TD, Housley MP, Boes M, Chen J, Kagnoff MF, Gillin FD et al. Central importance of immunoglobulin A in host defense against Giardia spp. Infect Immun 2002; 70(1): 11-18.

6. Lopez-Romero G, Quintero J, Astiazarán-García H, Velazquez C. Host defenses against Giardia lamblia. Parasite Immunol 2015; 37(8):394 406.

7. James AC, Amol Bhargava, Jose GF, Robin MY, Paul LB, Andre GB. Giardia duodenalis cathepsin B proteases degrade intestinal epithelial interleukin-8 and attenuate 
interleukin-8-induced neutrophil chemotaxis. Infect Immun 2014; 82(7):2772-2787.

8. Shukla G, Sidhu RK. Lactobacillus casei as a probiotic in malnourished Giardia lamblia-infected mice: a biochemical and histopathological study. Can J Microbiol 2011; 57(2):127-135.

9. Panaro MA, Cianciulli A, Mitolo V, Mitolo CI, Acquafredda A, Brandonisio 0 et al. Caspase-dependent apoptosis of the HCT-8 epithelial cell line induced by the parasite Giardia intestinalis. FEMS Immunol Med Microbiol 2007; 51(2): 302-309.

10. Lalle M. Giardiasis in the post genomic era: treatment, drug resistance and novel therapeutic perspectives. Infect Disord Drug Targets 2010; 10(4):283-294.

11. Reda AA. Probiotics for the control of helminth zoonosis. J Vet Med 2018; 9(6):4178986.

12. Amreen F. A review on probiotics: their potential impact on human health. EJPMR 2020; 7(6),189-199.

13. Shukla G, Sidhu RK, Verma A. Restoration of anthropometric, biochemical and histopathological alterations by Lactobacillus casei supplementation in Giardia intestinalis infected renourished balb/c mice. Antonie van Leeuwenhoek 2012; 102(1):61-72.

14. Bakhtiar SM, LeBlanc JG, Salvucci E, Ali A, Martin R, Langella $\mathrm{P}$ et al. Implications of the human microbiome in inflammatory bowel diseases. FEMS Microbiol Lett 2013; 342(1):10-17.

15. Mazroue AA, Elnokaly AA, El-lessy FM, Elbatrawy EN, Helal MM. Assessment of the therapeutic effect of probiotic Lactobacilli alone and in combination with metronidazole in murine giardiasis. Al-Azhar Uni J Virus Res Studies 2020; 2(1):1-18

16. Perrucci S, Fichi G, Ricci E, Galosi L, Lalle M, Rossi G. In vitro and ex vivo evaluation of the anti-Giardia duodenalis activity of the supernatant of Slab51 (Sivo Mixx). PLoS ONE 2019; 14(3): e0213385.

17. Nepelska M, Cultrone A, Béguet-Crespel F, Le Roux K, Doré J. Butyrate produced by commensal bacteria potentiates phorbol esters induced AP-1 response in human intestinal epithelial cells. PLoS ONE 2012; 7(12): e52869.

18. Davani-Davari D, Negahdaripour M, Karimzadeh I, Seifan M, Mohkam M, Masoumi SJ et al. Prebiotics: definition, types, sources, mechanisms, and clinical applications. Foods 2019; 8(3): 92.

19. Shukla G, Bhatia R, Sharma A. Prebiotic inulin supplementation modulates the immune response and restores gut morphology in Giardia duodenalis-infected malnourished mice. Parasitol Res 2016; 115(11):41894198.

20. Markowiak P, Zewska KS. Effects of probiotics, prebiotics, and synbiotics on human health. Nutrients 2017; 9(9):1021.

21. Shukla G, Sharma A, Bhatia R, Sharma M. Prophylactic potential of synbiotic (Lactobacillus casei and Inulin) in malnourished murine giardiasis: an immunological and ultrastructural study. Probiotics Antimicrob Proteins 2019; 11:165-174.

22. Garcia LS. Intestinal protozoa: flagellates and ciliates. Diagnostic Medical Parasitology $5^{\text {th }}$ ed. A.S.M press, Washington. D.C., Part II 2007; 771-812.
23. Dyab AK, Yones DA, Ibraheim ZZ, Hassan TM. Antigiardial therapeutic potential of dichloromethane extracts of Zingiber officinale and Curcuma longa in vitro and in vivo. Parasitol Res 2016; 115(7):2637-2645.

24. Abd El-Aziz TM, El-Beih NM, Soufy H, Naser S, Khalil FAM. Effect of Egyptian propolis on lipid profile and oxidative status in comparison with nitazoxanide in immunosuppressed rats infected with Cryptosporidium spp. Glob Vet 2014; 13 (1): 17-27.

25. Shukla G, Devi P, Sehgal R. Effect of Lactobacillus casei as a probiotic on modulation of giardiasis. Dig Dis Sci 2008; 53(10):2671-2679.

26. Benamrouz S, Guyot K, Gazzola S, Mouray A, Chassat T, Delaire B et al. Cryptosporidium parvum infection in SCID mice infected with only one oocyst: qPCR assessment of parasite replication in tissues and development of digestive cancer. PLOS ONE 2012; 7(12): 512-532.

27. Ross MH, Pawlina W. Histology: a text and atlas: with correlated cell and molecular biology. $7^{\text {th }}$ ed. Wolters Kluwer 2016; 984.

28. El-Kady AM, Abdel-Rahman IAM, Fouad SS, Allemailem KS, Istivan T, Ahmed SFM et al. Pomegranate peel extract is a potential alternative therapeutic for giardiasis. Antibiotics 2021; 10: 705 .

29. Engvall E, Perlmann P. Enzyme-linked immunosorbent assay (ELISA) quantitative assay of immunoglobulin G. Immunochemistry 1971; 8(9):871-874.

30. Humen MA, De Antoni GL, Benyacoub J, Costas ME, Cardozo MI, Kozubsky L et al. Lactobacillus johnsonii La1 antagonizes Giardia intestinalis in vivo. Infect Immun 2005; 73(2):1265-1269.

31. Shukla G, Sharma G, Goyal N. Probiotic characterization of Lactobacilli and yeast strains isolated from whey beverage and therapeutic potential of Lactobacillus yoghurt in murine giardiasis. Am J Biomed Sci 2010; 2: 248-261.

32. Amer EI, Mossallam SF, Mahrous H. Therapeutic enhancement of newly derived bacteriocins against Giardia lamblia. Exp Parasitol 2014; 146: 52-63.

33. Conlon MA, Bird AR. The impact of diet and lifestyle on gut microbiota and human health. Nutrients. 2015; 7(1): 17-44.

34. Ventura LLA, Oliveira DRD, Gomes MA, Torres MRF. Effect of probiotics on giardiasis. Where are we? Braz J Pharm Sci 2018; 54(2) :e17360.

35. Sand MM, El Maaty DA, Abd El-Aal NF, AL-Ghandour A, Farag A, Yousef A et al. Theraputic efficacy of probiotics against experimental giardiasis: histopathological, histochemical and ultrastructural changes. JESP 2020; 50(1):21-32.

36. PatelR, Dupont HL. New approachesfor bacteriotherapy: prebiotics, new-generation probiotics, and symbiotics. Clin Infect Dis 2015; 60(2): S108-121.

37. Hawrelak J. Giardiasis: pathophysiology and management. Altern Med Rev 2003; 8:129-142.

38. Kelesidis T, Pothoulakis C. Efficacy and safety of the probiotic Saccharomyces boulardii for the prevention and therapy of gastrointestinal disorders. Therap Adv Gastroenterol 2012; 5(2): 111-125. 
39. Shukla G, Kaur H, Sharma L. Comparative therapeutic effect of probiotic Lactobacillus casei alone and in conjunction with antiprotozoal drugs in murine giardiasis. Parasitol Res 2013; 112(6): 2143-2149.

40. Di Santo N, Ehrisman J. Research perspective: potential role of nitazoxanide in ovarian cancer treatment. Old drug, new purpose? Cancers (Basel) 2013; 5(3):11631176.

41. Galeh TM, Kazemi A, Mahami-Oskouei M, Baradaran B, Spotin A, Sarafraz S et al. Introducing nitazoxanide as a promising alternative treatment for symptomatic to metronidazole-resistant giardiasis in clinical isolates. Asian Pac J Trop Med 2016; 9(9): 887-892.

42. Kamda JD, Nash TE, Singer SM. Giardia duodenalis: Dendritic cell defects in IL- 6 deficient mice contribute to susceptibility to intestinal infection. Exp Parasitol 2012; 130: 288-291.

43. Jimenez JC, Fontaine J, Creusy C, Fleurisse L, Grzych JM, Capron M, et al. Antibody and cytokine responses to Giardia excretory/secretory proteins in Giardia intestinalis-infected BALB/c mice. Parasitol Res 2014; 113:2709-2718.

44. Khader SA, Gaffen SL, Kolls JK. Th17 cells at the crossroads of innate and adaptive immunity against infectious diseases at the mucosa. Mucosal Immunol 2009; 2:403411.

45. Scheller J, Chalaris A, Schmidt-Arras D, Rose-John S. The pro- and anti-inflammatory properties of the cytokine interleukin 6. Biochim Biophys Acta 2011; 1813:878- 888.

46. Kimura A, Naka T, Kishimoto T. IL-6- dependent and -independent pathways in the development of interleukin 17-producing T helper cells. Proc Natl Acad Sci USA 2007; 104: 12099-12104.

47. Bienz M, Dai WJ, Welle M, Gottstein B, Müller N. Interleukin-6-deficient mice are highly susceptible to Giardia lamblia infection but exhibit normal intestinal immunoglobulin A responses against the parasite. Infect Immun 2003; 71:1569-1573.

48. Galdeano CM, Perdigon G. The probiotic bacterium Lactobacillus casei induces activation of the gut mucosal immune system through innate immunity. Clin Vaccine Immunol 2006; 13(2): 219-226.

49. Goyal N, Shukla G. Probiotic Lactobacillus rhamnosus GG modulates the mucosal immune response in Giardia intestinalis infected BALB/c mice. Dig Dis Sci 2013; 58(5):1218-1225. 